\title{
Screening for Depressive Disorder in Elderly Patients with Chronic Physical Diseases Using the Patient Health Questionnaire-9
}

\author{
Seon-Cheol Park ${ }^{1}$, Hwa-Young Lee ${ }^{2}$, Dong-Woo Lee ${ }^{3}$, Sang-Woo Hahn', Sang-Ho Park ${ }^{5}$, Yeo Ju Kim ${ }^{6}$, \\ Jae Sung $\mathrm{Choi}^{7}$, Ho-Sung Lee ${ }^{7}$, Soyoung Irene $\mathrm{Lee}^{8}$, Kyoung-Sae $\mathrm{Na}^{9}$, Sung Won Jung ${ }^{10}$, Se-Hoon Shim², \\ Ki Won Kim ${ }^{11,12}$, Jong-Woo Paik ${ }^{13}$, and Young-Joon Kwon ${ }^{2}$ \\ ${ }^{1}$ Department of Psychiatry, Inje University College of Medicine and Haeundae Paik Hospital, Busan, Republic of Korea \\ ${ }^{2}$ Department of Psychiatry, Soonchunhyang University Cheonan Hospital, Cheonan, Republic of Korea \\ ${ }^{3}$ Department of Psychiatry, Inje University Sanggye Paik Hospital, Seoul, Republic of Korea \\ ${ }^{4}$ Department of Psychiatry, Soonchunhyang University Seoul Hospital, Seoul, Republic of Korea \\ ${ }^{5}$ Department of Cardiology, Soonchunhyang University Cheonan Hospital, Cheonan, Republic of Korea \\ ${ }^{6}$ Department of Endocrinology, Soonchunhyang University Cheonan Hospital, Cheonan, Republic of Korea \\ ${ }^{7}$ Department of Pulmonology and Allergy, Soonchunhyang University Cheonan Hospital, Cheonan, Republic of Korea \\ ${ }^{8}$ Department of Psychiatry, Soonchunhyang University Bucheon Hospital, Bucheon, Republic of Korea \\ ${ }^{9}$ Department of Psychiatry, Gachon University School of Medicine, Incheon, Republic of Korea \\ ${ }^{10}$ Department of Psychiatry, Keimyung University Dongsan Medical Center, Daegu, Republic of Korea \\ ${ }^{11}$ National Institute of Dementia, Seongnam, Republic of Korea \\ ${ }^{12}$ Department of Neuropsychiatry, Seoul National University Bundang Hospital, Seongnam, Republic of Korea \\ ${ }^{13}$ Department of Psychiatry, Kyung Hee University School of Medicine, Seoul, Republic of Korea
}

Objective We aimed to identify depressive symptom profiles that indicated the presence of depressive disorder and present optimal cutoff sub-scores for depressive symptom profiles for detecting depressive disorder in elderly subjects with chronic physical diseases including diabetes, chronic obstructive pulmonary disease/asthma, and coronary artery disease, using the Patient Health Questionnaire-9 (PHQ-9).

Methods Two hundred and thirty-one elderly patients with chronic physical diseases were recruited consecutively from a university-affiliated general hospital in South Korea.

Results Greater severities of all 9 depressive symptoms in the PHQ-9 were presented in those with depressive disorder rather than those without depressive disorder. A binary logistic regression modeling presented that little interest [adjusted odds ratio $(\mathrm{aOR})=4.648, \mathrm{p}<0.001$ ], reduced/increased sleep $(\mathrm{aOR}=3.269, \mathrm{p}<0.001)$, psychomotor retardation/agitation $(\mathrm{aOR}=2.243, \mathrm{p}=0.004)$, and concentration problem $(\mathrm{aOR}=16.116, \mathrm{p}<0.001)$ were independently associated with increased likelihood of having depressive disorder. Receiver operating characteristics (ROC) curve analysis presented that the optimal cut-off value of score on the items for little interest, reduced/increased sleep, psychomotor retardation/agitation and concentration problem (PHQ-9) for detecting depressive disorder was 4 with $61.9 \%$ of sensitivity and $91.5 \%$ of specificity [area under curve (AUC) $=0.937, \mathrm{p}<0.001$ ].

Conclusion Our findings suggested that the diagnostic weighting of little interest, reduced/increased sleep, psychomotor retardation/ agitation, and concentration problem is needed to detect depressive disorder among the elderly patients with chronic physical diseases.

Psychiatry Investig 2017;14(3):306-313

Key Words Depressive disorder, Elderly, Chronic physical diseases, Patient health questionnaire-9.

\section{INTRODUCTION}

In medical populations, the continuum of depression can

Received: April 12, 2016 Revised: May 22, 2016

Accepted: June 9, 2016 Available online: March 14, 2017

$\triangle$ Correspondence: Hwa-Young Lee, MD, PhD

Department of Psychiatry, Soonchunhyang University Cheonan Hospital, 31 Suncheonhyang 6-gil, Dongnam-gu, Cheonan 31151, Republic of Korea Tel: +82-41-570-3876, Fax: +82-41-592-3804, E-mail: leehy@schmc.ac.kr

@ This is an Open Access article distributed under the terms of the Creative Commons Attribution Non-Commercial License (http://creativecommons.org/licenses/bync/4.0) which permits unrestricted non-commercial use, distribution, and reproduction in any medium, provided the original work is properly cited. range from nonpathological sadness and grief to depressive disorders specified in the Diagnostic and Statistical Manual of Mental Disorders, 5th Edition (DSM-5). The depressive disorders specified in the DSM-5 include major depressive disorder (MDD), dysthymia, premenstrual dysphoric disorder, depressive disorder due to another medical condition, and other specified depressive disorders. ${ }^{1}$ Prevalence of major depressive disorder, minor depressive disorder, and overall late-life depression were $5.37 \%, 5.52 \%$, and $10.89 \%$, respectively in the elderly population in a nationwide survey of South Korea. ${ }^{2}$ 
Geriatric patients with depression predominantly presented with atypical clinical features and this often hampered the detection of depression. The age-specific manifestations of geriatric depression are regarded as the important clinical concerns; and its manifestations are characterized by hypochondriasis and somatic preoccupation associated anxiety and insomnia rather than typical features including melancholia/persistent sadness. ${ }^{3}$ In addition, an epidemiological study showed that, in patients with major cognitive disorder, the prevalence of depressive disorder and major depressive disorder were 11$51 \%$ and $15-20 \%$, respectively. A theoretical speculation is that geriatric depression is continuous with minor cognitive impairment and major depressive disorder and can be regarded as a prodromal state of major cognitive disorder. ${ }^{4}$ A systemic review and meta-analysis demonstrated that disability, bereavement, sleep disturbance, female gender and others are identified as important risk factors for depressive disorder in elderly community subjects. ${ }^{5}$ Another review showed that biological factors are regarded as more important predictors for development of geriatric depression. ${ }^{6}$ Thus, chronic physical diseases are regarded as possible risk factors for depressive disorder. $^{7}$

The relationship between depressive disorder and physical diseases can be bidirectional and complex. In selected medical diseases, prevalence of major depressive disorder is as follows: $0-38 \%$ for cancer, ${ }^{8} 9-28 \%$ for diabetes, ${ }^{9} 17-27 \%$ for heart disease, ${ }^{10} 20-50 \%$ for chronic obstructive pulmonary disease (COPD)/asthma, ${ }^{11}$ 5-20\% for HIV/AIDS, ${ }^{12} 14-19 \%$ for stroke, ${ }^{13} 20-55 \%$ for epilepsy, ${ }^{14} 40-60 \%$ for multiple sclerosis, ${ }^{15}$ $30-50 \%$ for Alzheimer's disease, ${ }^{16}$ and $4-75 \%$ for Parkinson's diseases. ${ }^{17}$ A national health survey of 30,801 adults in the US reported that the prevalence of depressive disorder among adults with one, two, and three chronic physical diseases was $7.7 \%, 9.8 \%$, and $12 \%$, respectively, whereas the prevalence of depressive disorder among adults without chronic physical diseases was $4.7 \%$. There is a positive relationship trend between the number of chronic physical diseases and risk of depressive disorder. ${ }^{18}$ In addition, co-incidence of depressive disorder and chronic physical diseases can contribute to poor prognosis and increased mortality. ${ }^{7}$ Moreover, since medically ill patients often show fatigue, anorexia, weight loss, retardation, reduced concentration, loss of interest, and desire to die independent of co-morbid depression, evaluating the presence or absence of depression might be complicated. ${ }^{19,20}$ Hence, detection and management of depressive disorder in the elderly patients with chronic physical diseases can be an important clinical issue in the realm of consultation-liason psychiatry or psychosomatic medicine. In addition, depressive symptom profiles indicating the presence of depressive disorder need to be identified to efficiently detect depressive disorder in elderly patients with chronic physical diseases. ${ }^{21}$

As described elsewhere, ${ }^{21}$ in the context of developing a general hospital-based model for early detection of depressive disorder among elderly patients with chronic medical disease, we aimed to 1) present the prevalence of depressive disorders, 2) determine depressive symptom profiles that indicated the presence of depressive disorders, and 3) present optimal cutoff sub-scores for depressive symptom profiles for detecting depressive disorder in elderly subjects with chronic physical diseases including diabetes mellitus (DM), COPD/asthma, and coronary artery disease (CAD).

\section{METHODS}

\section{Study subjects and procedures}

From September 2012 to February 2013, the study subjects were consecutively recruited from among elderly outpatients of the departments of endocrinology, pulmonology, and cardiology in a university-affiliated general hospital in South Korea. The inclusion criteria of our study were as follows: 1) age $\geq 60$ years and 2) diagnosis of DM (E10-E14), COPD/asthma (J40-J47), or CAD (I20-I25), confirmed with the International Classification of Diseases and Related Health Problems, 10th revision (ICD-10) ${ }^{22}$ by specialists consisting of endocrinologist, pulmonologist, or cardiologist, respectively. The exclusion criteria of our study were as follows: 1) prior history or current episode of any psychiatric disorders, including intellectual disability, schizophrenia, bipolar disorder, depressive disorder, and others, 2) seizure and other neurological disorders, 3) complications of cerebrovascular accidents, 4) severe medical diseases that entirely hinder the study participation, and 5) illiteracy. Study procedures consisted of self-questionnaires for sociodemographic and clinical characteristics and semi-structured interview for detecting depressive disorder. Our study was approved by the Institutional Review Board of Soonchunhyang University Cheonan Hospital (receipt number: SCHCA_IRB_2012-57). All study subjects provided written informed consent before the initiation of study initiation. Although there were 292 eligible patients, 61 (20.9\%) refused to participate in our study. We recruited a final sample of 231 elderly patients with chronic physical diseases.

\section{Sociodemographic and clinical characteristics}

The data for age (years), gender, marriage (bereaved or divorced; married), living status (living alone; living together), educational attainment (below middle school education; above high school education), occupation (unemployed; employed), and family history of depressive disorder (present; absent) were collected. 


\section{Assessment tools}

The Hamilton Depression Rating Scale (HAMD) ${ }^{23}$ which was initially developed to evaluate disease severity and consists of 17 items in the context of semi-structured interview, was used to detect depressive disorder and define severity classification in the subjects. Yi et al. ${ }^{24}$ formally translated the HAMD into Korean and performed standardization of its Korean version (internal consistency with Cronbach's alpha $=0.76$; interrater reliability with $\mathrm{r}=0.94$ and $\mathrm{p}<0.001$ ). In our study, the HAMD was performed by a research coordinator, supervised by a specialized psychiatrist about geriatric depression. According to the proposal of Zimmerman et al., ${ }^{25}$ the HAMD cut-off value for identifying depressive disorder was 8 . In addition, severity classifications were defined as follows: $0-7$ for no depression, 8-16 for mild depression, 17-23 for moderate depression, and $\geq 24$ for severe depression, respectively.

The Patient Health Questionnaire-9 (PHQ-9), ${ }^{26}$ which is a self-questionnaire consisting of 9 depressive symptoms in the diagnostic criteria for major depressive disorder in the Diagnostic and Statistical Manual of Mental Disorders, 4th edition (DSM-IV), ${ }^{27}$ was used to evaluate the severity of each of depressive symptoms. Park et al. ${ }^{28}$ formally translated the PHQ-9 into Korean and performed standardization of its Korean version (internal consistency with Cronbach's alpha $=0.81$; test-retest reliability with $r=0.89$ and $p<0.01$ ). Further, the PHQ-9 has been proposed as a valuable tool for evaluating depressive symptoms among elderly persons with medical illness in a primary health care setting. Therefore, we used the PHQ-9 to identify symptom profiles indicating the presence of depressive disorder. ${ }^{29}$

The Mini-Mental Status Examination (MMSE), ${ }^{30}$ which is a component of the Consortium to Establish a Registry for Alzheimer's Disease (CERAD), was used to evaluate cognitive function. Lee et al. ${ }^{31}$ formally translated the MMSE into Korean and performed its standardization [internal consistency with Cronbach's alpha $=0.92$; validity with $\mathrm{F}(1,287)=356.3$ and $\mathrm{p}<0.0001]$. Score on the MMSE of $\leq 24$ was defined as cognitive impairment.

The Charlson Comorbidity Index (CCI ${ }^{32}$ was used to evaluate severity of chronic physical diseases, including DM, COPD/ asthma, CAD, and other comorbid physical diseases. The CCI was categorized into $0,1,2$, and $3+$ groups by adjusting the total score on the rating from 1 to 6 in terms of 19 physical diseases. The ICD-10 version of the CCI was standardized.

\section{Statistical analysis}

Sociodemographic and clinical characteristics (including, severity of depressive symptoms) were compared between the subjects with and without depressive disorder using independent $t$-test for continuous variables and $\chi^{2}$ test for dis- crete variables. To adjust the potential effects of confounding variables, analysis of covariance (ANCOVA) for continuous variables and logistic regression analysis for discrete variables were used. A binary logistic regression model was constructed to identify depressive symptoms associated with increased likelihood of depressive disorder, while adjusting for the potential effects of confounding variables. Dependent variable was defined as the presence or absence of depressive disorder, whereas covariates were defined as the severity of nine depressive symptoms (PHQ-9). In the model, goodness of fit to control interaction and forward selection method to avoid multicolinearity was used. The exploratory receiver operating characteristics (ROC) curve analyses was used to determine the optimal cut-off sub-score for depressive symptom profiles (PHQ-9) that distinguished between the presence or absence of depressive disorder. . As described elsewhere, ${ }^{33,34}$ the ROC curve analyses were developed from signal-detection theory. To present the optimal cut-off value with appropriate false positives and false negatives, the sensitivity/specificity pairs for all possible threshold levels were considered. Significance was set at $\mathrm{p}<0.01$ (two-tailed) for all tests, for the purpose of reducing the family wise error due to multiple comparisons. All statistical analyses were performed using SPSS 21 for Windows (SPSS Inc., Chicago, IL, USA).

\section{RESULTS}

\section{Prevalence of depressive disorder and classification of its severity}

Forty-two subjects (18.2\%) were found to have depressive disorder, as they scored $\geq 8$ on the HAMD. ${ }^{26}$ The overall distribution of the HAMD tended to be unimodal, positively skewed (skewness $=1.9$ ) with $67.1 \%$ of the subjects scoring $<5$, and leptokurtic (kurtosis=5.1) with $13.4 \%$ scoring 4 . The mean score on the HAMD was $4.3(\mathrm{SD}=4.3$; range $=0-27)$. Among 42 subjects with depressive disorder, $88.1 \%(\mathrm{n}=37)$ were estimated to mild depression, $9.5 \%(\mathrm{n}=4)$ to moderate depression, and $2.4 \%(\mathrm{n}=1)$ to severe depression, respectively.

\section{Comparison of baseline characteristics between the subjects with and without depressive disorder}

As shown in Table 1, the subjects with depressive disorder were significantly higher proportion of women $\left(X^{2}=12.555\right.$, $\mathrm{p}<0.0001)$ and living alone $\left(\chi^{2}=8.459, \mathrm{p}=0.004\right)$ than those without depressive disorder. In addition, although the statistical values were not significant, those with depressive disorder tended to have higher proportion of bereaved or divorced status $\left(\chi^{2}=3.923, p=0.048\right)$ and lower score on the MMSE $(t=-2.506, p=0.13)$. Thus, further statistical analyses were performed with adjusting the effects of gender, marital status, 
living status, and score on the MMSE. There were no significant differences in age $[\mathrm{F}(1,226)=0.893, \mathrm{p}=0.346]$, employment [adjusted odds ratio $(\mathrm{aOR})=0.870, \mathrm{p}=0.737$ ], education $(\mathrm{aOR}=0.633, \mathrm{p}=0.504)$, enrolled department $\left(\chi^{2}=1.253, \mathrm{p}=\right.$ 0.534), DM (aOR=0.434, $\mathrm{p}=0.036), \mathrm{COPD} / \mathrm{asthma}(\mathrm{aOR}=$ 1.583, $\mathrm{p}=0.238), \mathrm{CAD}(\mathrm{aOR}=5.111, \mathrm{p}=0.126), \mathrm{CCI}\left(\chi^{2}=0.877\right.$, $\mathrm{p}=0.831)$, family history of depressive disorder $(\mathrm{aOR}=1.907$, $\mathrm{p}=0.557)$, and cognitive impairment $(\mathrm{aOR}=0.732, \mathrm{p}=0.536)$ between those with and without depressive disorder.

\section{Comparison of depressive symptom profiles between the subjects with and without depressive disorder}

As shown in Table 2, with adjusting the effects of gender, marital status, living status, and score on MMSE, those with

Table 1. Baseline characteristics of the subjects with and without depressive disorder

\begin{tabular}{|c|c|c|c|c|c|c|}
\hline Characteristics & $\begin{array}{c}\text { Total samples } \\
(\mathrm{N}=231)\end{array}$ & $\begin{array}{c}\text { Depression } \\
(\mathrm{N}=42)\end{array}$ & $\begin{array}{c}\text { No depression } \\
\quad(\mathrm{N}=189)\end{array}$ & $\begin{array}{c}\text { Statistical } \\
\text { coefficient }\end{array}$ & $\begin{array}{l}\text { Unadjusted } \\
\text { p value }\end{array}$ & $\begin{array}{l}\text { Adjusted } \\
\text { p value* }\end{array}$ \\
\hline Age, mean (SD) years & $73.1(5.7)$ & $72.9(5.5)$ & $73.1(5.8)$ & $\mathrm{t}=-0.203$ & 0.839 & 0.374 \\
\hline Female, N (\%) & $108(46.8)$ & $30(71.4)$ & $78(41.3)$ & $\chi^{2}=12.555$ & $<0.0001$ & - \\
\hline Bereaved or divorced, N (\%) & $171(74.0)$ & $26(61.9)$ & $145(76.7)$ & $\chi^{2}=3.923$ & 0.048 & - \\
\hline Living alone, $\mathrm{N}(\%)$ & $33(14.3)$ & $12(28.6)$ & $21(11.2)$ & $\chi^{2}=8.459$ & 0.004 & - \\
\hline Unemployed, N (\%) & $149(64.5)$ & $31(73.8)$ & $118(79.2)$ & $\chi^{2}=1.942$ & 0.163 & 0.737 \\
\hline Below middle school education, N (\%) & $193(83.5)$ & $39(92.9)$ & $154(81.5)$ & $\chi^{2}=3.235$ & 0.072 & 0.504 \\
\hline Enrolled department, N (\%) & & & & $\chi^{2}=1.253$ & 0.534 & - \\
\hline Endocrinology & $89(38.5)$ & $13(31.0)$ & $76(40.2)$ & & & \\
\hline Pulmonology & $92(39.8)$ & $19(45.2)$ & $73(38.6)$ & & & \\
\hline Cardiology & $50(21.6)$ & $10(23.8)$ & $40(21.2)$ & & & \\
\hline Diabetes mellitus, $\mathrm{N}(\%)$ & $97(42.0)$ & $13(31.0)$ & $84(44.4)$ & $\chi^{2}=2.568$ & 0.109 & 0.036 \\
\hline COPD/asthma, N (\%) & $98(42.4)$ & $20(47.6)$ & $78(41.3)$ & $\chi^{2}=0.567$ & 0.451 & 0.238 \\
\hline Congestive heart failure, $\mathrm{N}(\%)$ & $80(34.6)$ & $18(42.9)$ & $62(32.8)$ & $\chi^{2}=1.534$ & 0.216 & 0.126 \\
\hline CCI, N (\%) & & & & $\chi^{2}=0.877$ & 0.831 & - \\
\hline One & $70(30.4)$ & $15(35.7)$ & $55(29.3)$ & & & \\
\hline Two & $72(31.3)$ & $12(28.6)$ & $60(31.9)$ & & & \\
\hline Three or more & $87(37.8)$ & $15(35.7)$ & $73(38.8)$ & & & \\
\hline Family history of depression, $\mathrm{N}(\%)$ & $12(5.2)$ & $1(2.4)$ & $11(5.8)$ & $\chi^{2}=0.825$ & 0.364 & 0.557 \\
\hline MMSE, mean (SD) & $22.9(4.3)$ & $21.6(4.4)$ & $23.4(4.1)$ & $\mathrm{t}=-2.506$ & 0.013 & - \\
\hline Cognitive impairment, $\mathrm{N}(\%)$ & $74(32.4)$ & $15(36.6)$ & $59(31.6)$ & $\chi^{2}=0.389$ & 0.533 & 0.536 \\
\hline
\end{tabular}

*adjusted for the effects of gender, marital status, living status, and score on the MMSE. CCI: Charlson comorbidity index, COPD: chronic obstructive pulmonary disease, MMSE: mini-mental status examination

Table 2. Score on the Patient Health Questionnaire-9 of the subjects with and with depressive disorder

\begin{tabular}{|c|c|c|c|c|c|c|}
\hline Questionnaire items & $\begin{array}{c}\text { Total samples } \\
(\mathrm{N}=231)\end{array}$ & $\begin{array}{c}\text { Depression } \\
(\mathrm{N}=42)\end{array}$ & $\begin{array}{l}\text { No depression } \\
\qquad(\mathrm{N}=189)\end{array}$ & $\begin{array}{l}\text { Statistical } \\
\text { coefficient }\end{array}$ & $\begin{array}{c}\text { Unadjusted } \\
\text { p value }\end{array}$ & $\begin{array}{l}\text { Adjusted } \\
\text { p value* }\end{array}$ \\
\hline Depressed mood, mean (SD) & $0.6(0.8)$ & $1.2(1.0)$ & $0.4(0.7)$ & $\mathrm{t}=6.128$ & $<0.0001$ & $<0.0001$ \\
\hline Little interest, mean (SD) & $0.4(0.7)$ & $1.1(0.9)$ & $0.3(0.5)$ & $t=6.130$ & $<0.0001$ & $<0.0001$ \\
\hline Reduced/increased sleep, mean (SD) & $0.8(1.0)$ & $1.8(1.1)$ & $0.6(0.8)$ & $\mathrm{t}=6.954$ & $<0.0001$ & $<0.0001$ \\
\hline Reduced/increased appetite, mean (SD) & $0.7(0.9)$ & $1.6(1.1)$ & $0.5(0.8)$ & $t=6.690$ & $<0.0001$ & $<0.0001$ \\
\hline Psychomotor retardation/agitation, mean (SD) & $0.4(0.8)$ & $1.2(1.0)$ & $0.3(0.7)$ & $\mathrm{t}=5.827$ & $<0.0001$ & $<0.0001$ \\
\hline Feeling tired, mean (SD) & $0.2(0.5)$ & $0.7(0.8)$ & $0.1(0.3)$ & $\mathrm{t}=4.338$ & $<0.0001$ & $<0.0001$ \\
\hline Guilty feeling, mean (SD) & $0.2(0.6)$ & $0.7(0.9)$ & $0.1(0.4)$ & $\mathrm{t}=3.586$ & $<0.0001$ & $<0.0001$ \\
\hline Concentration problem, mean (SD) & $0.2(0.5)$ & $0.6(0.8)$ & $0.1(0.3)$ & $\mathrm{t}=4.300$ & $<0.0001$ & $<0.0001$ \\
\hline Suicidal ideation, mean (SD) & $0.2(0.4)$ & $0.5(0.6)$ & $0.1(0.3)$ & $\mathrm{t}=3.857$ & $<0.0001$ & $<0.0001$ \\
\hline Total score, mean (SD) & $3.8(3.9)$ & $9.5(4.4)$ & $2.5(2.4)$ & $t=9.928$ & $<0.0001$ & $<0.0001$ \\
\hline
\end{tabular}

*adjusted for the effects of gender, marital status, living status, and score on the MMSE. MMSE: mini-mental status examination 
Table 3. Binary logistic regression model to identify the depressive symptom profiles indicating depressive disorder ${ }^{\dagger}$

\begin{tabular}{lcccccc}
\hline \multicolumn{1}{c}{ Depressive symptom } & $\mathrm{B}$ & Standard error & Wald & Adjusted p value* & Adjusted OR* & 95\% CI \\
\hline Little interest & 1.536 & 0.422 & 13.248 & $<0.001$ & 4.648 & $2.032-10.631$ \\
Reduced/increased sleep & 1.185 & 0.289 & 16.828 & $<0.001$ & 3.269 & $1.856-5.757$ \\
Psychomotor retardation/agitation & 0.808 & 0.283 & 8.155 & 0.004 & 2.243 & $1.288-3.906$ \\
Concentration problem & 2.780 & 0.723 & 14.803 & $<0.001$ & 16.116 & $3.911-66.414$ \\
\hline
\end{tabular}

*adjusted for the effects of gender, marital status, living status, and score on the MMSE, ${ }^{\dagger}$ depressive mood, reduced/increased appetite, feeling tired, guilty feeling, and suicidal ideation were not included in the binary logistic regression model. CI: confidence interval, OR: odds ratio

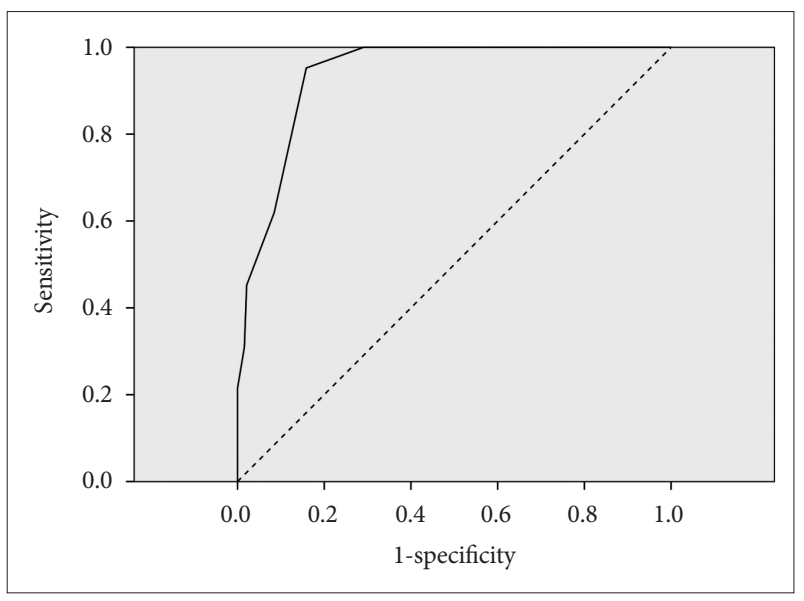

Figure 1. Receiver operating characteristics (ROC) curves for total score on the four items* of the PHQ-9 in detecting depressive disorder. *consisted of the items for the little interest, reduced/increased sleep, psychomotor retardation/agitation and concentration problem. PHQ-9: Patient Health Questionnaire-9.

depressive disorder had greater scores on all the depressive symptoms (PHQ-9), consisting of depressive mood [F (1, $226)=37.786, p<0.0001]$, little interest $[\mathrm{F}(1,226)=64.540$, $\mathrm{p}<0.0001]$, reduced/increased sleep $[\mathrm{F}(1,226)=52.833, \mathrm{p}<$ $0.0001]$, reduced/increased appetite $[\mathrm{F}(1,226)=55.267, \mathrm{p}<$ $0.0001]$, psychomotor retardation/agitation $[\mathrm{F}(1,226)=44.259$, $\mathrm{p}<0.0001]$, feeling tired $[\mathrm{F}(1,226)=44.652, \mathrm{p}<0.0001]$, guilty feeling $[\mathrm{F}(1,226)=32.718, \mathrm{p}<0.0001]$, concentration problem $[\mathrm{F}(1,226)=74.506, \mathrm{p}<0.0001]$, and suicidal ideation [F (1, $226)=22.632, \mathrm{p}<0.0001]$, than those without depressive disorder. In addition, those with depressive disorder had higher total score on the PHQ-9 [F $(1,226)=323.540, \mathrm{p}<0.0001]$ than those without depressive disorder.

\section{Binary logistic regression model to identify the depressive symptom profiles indicating depressive disorder}

As shown in Table 3, binary logistic regression modeling was fitted to identify depressive symptom profiles indicating the presence of depressive disorder. The Hosmer-Lemeshow goodness-of-fit test $\left(\chi^{2}=1.338, \mathrm{df}=8\right.$, and $\left.\mathrm{p}=0.995\right)$ confirmed the acceptability of the binary logistic regression modeling. After adjusting for the effects of gender, marital status, living status, and MMSE score, the final model explained 68.1\% (Nagelkerke $\mathrm{R}^{2}$ ) of the variability of depressive disorder and showed that little interest $(\mathrm{aOR}=4.648, \mathrm{p}<0.0001)$, reduced/increased sleep $(\mathrm{aOR}=3.269, \mathrm{p}<0.0001)$, psychomotor retardation/agitation $(\mathrm{aOR}=2.243, \mathrm{p}=0.004)$, and concentration problem $(\mathrm{aOR}=16.116, \mathrm{p}<0.0001)$ were independently associated with increased likelihood of presence of depressive disorder.

\section{ROC curve analysis for score on the several items of the PHQ-9 to detect depressive disorder}

As shown in Figure 1, the optimal cut-off values of scores for the items of little interest, reduced/increased sleep, psychomotor retardation/agitation and concentration problem (PHQ9) for detecting the presence of depressive disorder was four with a sensitivity value of $61.9 \%$ and a specificity value of 91.5\% [area under curve (AUC) $=0.937, \mathrm{p}<0.0001$ ].

\section{DISCUSSION}

In summary, among 231 elderly subjects with chronic physical diseases (DM, COPD/asthma, and CAD), 18.2\% were estimated as depressive disorder with $\geq 8$ score on the HAMD. With adjusting the effects of gender, marital status, living status, and score on the MMSE, those with depressive disorder presented higher scores on all the items of the PHQ-9 than those without depressive disorder; and binary logistic regression modeling showed that little interest, reduced/increased sleep, psychomotor retardation/agitation, and concentration problem were independently associated with the depressive disorder. ROC curve analysis results indicated that the optimal cut-off value of the 4 items (little interest, reduced/increased sleep, psychomotor retardation/agitation, and concentration problem; PHQ-9) was 4 with $61.9 \%$ of sensitivity and $91.5 \%$ of specificity.

As mentioned earlier, the prevalence of major depressive disorder in medical diseases is reported as follows: 9-28\% for diabetes, ${ }^{9} 17-27 \%$ for heart disease, ${ }^{10}$ and $20-50 \%$ for chronic obstructive pulmonary disease (COPD)/asthma. ${ }^{11}$ Hence, the $18.2 \%$ prevalence of depressive disorder $(\geq 8$ score on the HAMD) in our study was partly consistent with previous findings, but regarded as a relative low level. Since the HAMD 
was developed before establishment of the DSM diagnostic construct for depressive disorders, it differs from the DSM approach and is characterized by favoring the association with somatic presentations including the insomnia, psychomotor retardation, loss of appetite, loss of energy, loss of weight, and loss of libido. ${ }^{35}$ The HAMD might be an adequate assessment tool for the evaluation of hypochondriasis, somatic concern, and other age-specific manifestations of geriatric depression. However, the time needed to complete the assessment tools and the complexity of questions can influence the acceptability of depression screening. ${ }^{36}$ Screening for depression might be hampered by the application of the HAMD, which is a long semi-structured interview, taking 15 to 20 minutes. ${ }^{37}$ In addition, in diabetic patients, female gender, lack of social support, younger age, low socioeconomic status, occurrence of late complications, poor glycemic control, and hypoglycemia are regarded as the risk factors for depression..$^{38}$ In patients with cardiovascular disease, anxiety and quality of life overlap with depression and are regarded as the predictors for poor outcome..$^{39}$ In asthma patients, dyspnea and sleep problems are significantly associated with depression. ${ }^{40}$ Hence, the relatively low prevalence of depressive disorder in our study, was attributable to the conditions that are unique to elderly patients; moreover, the effects of other sociodemographic variables including socioeconomic status were not adjusted. In addition, although the CCI was used, specific clinical trajectories and outcomes of comorbid physical diseases were not considered in our study.

Significantly greater proportion of women who were living alone and tendency towards greater proportion among bereaved or divorced in the subjects with depressive disorder were consistent with previous findings. A systemic review and meta-analysis indicated that bereavement, sleep problems, disability, prior depression, and female gender can be regarded as the risk factors for depressive disorder among the elderly subjects. ${ }^{6}$ In addition, there is a trend towards lower score on the MMSE, among elderly subjects with depressive disorder. The trend is partly consistent with the speculations that cognitive impairment is often coupled with geriatric depression and depression might be a prodromal state of major cognitive disorder in the elderly subjects. ${ }^{41}$

Greater scores on all the 9 depressive symptoms of the PHQ-9 were presented in those with depressive disorder. In addition, the 4 depressive symptoms consisting of little interest, reduced/increased sleep, psychomotor retardation/agitation, and concentration problem were identified as independent covariates for the presence of depressive disorder in the binary logistic regression modeling. A study on symptom profiles of late-life depression showed that psychomotor change is associated with the vascular and degenerative indicators, concentration disturbance is associated with the degenerative indicator, and little interest and sleep disturbance are associated with the inflammatory indicator, respectively. ${ }^{42}$ Hence, the 4 depressive symptoms can correspond to the characterized features of geriatric depression, which might reflect the predominant underlying pathogenic mechanism. Our findings are consistent with age-specific symptom preponderance of geriatric depression (hypochondriasis and somatic concerns rather than melancholia and anhedonia), ${ }^{5}$ and provide the clinical implications for detecting depressive disorder among the elderly patients with chronic physical diseases. Hence, it is speculated that diagnostic weightings of the 4 depressive symptoms are needed in the detection of depressive disorder among elderly subjects with chronic physical diseases (DM, COPD/asthma, and CAD). A ROC curve analysis identified the optimal cut-off value of score on the 4 depressive symptoms to detect depressive disorder was 4 with $61.9 \%$ of sensitivity and $91.5 \%$ of specificity. Four of the score on the 4 depressive symptoms of the PHQ-9 can have limitation of relatively high rate of false-positive rather than false-negative, and mask the depressive conditions from presence of 4 symptoms for 2 to 3 day to presence of 2 symptoms for nearly every day in the period of 2 weeks.

There were several limitations to our study. Firstly, the specific clinical characteristics of DM, COPD/asthma, and CAD were not considered, although the comorbid medical diseases were evaluated with the CCI. Secondly, acceptability of the HAMD to detect depressive disorder can be limited, since the HAMD requires considerable time to complete the assessment tools and its questions can be relatively complex. Thirdly, more elaborated assessment tools for cognitive domains were not used, although the MMSE was used to evaluate cognitive functions in our study. Fourthly, assessment tools covering anxiety, quality of life, economic factors, and other various variables were not used in our study. Thus, considering the depressive disorder among the elderly patients with chronic physical diseases, further discussion about various psychopathology and psychosocial factors were limited. Finally, since the HAMD has included several items for somatic symptoms, using it to evaluate depressive symptoms in medically ill patients is controversial. ${ }^{43,44}$ In addition, there has been a proposal that the Geriatric Depression Scale (GDS) could be more useful than HAMD to detect depressive disorder in this population. ${ }^{45}$ However, Koenig and Kuchibhatla ${ }^{46}$ presented that among 502 hospitalized medically ill elderly patients with depression, the HAMD score predicted several clinical indicators including the number of days spent in hospital in the past year, the number of days spent in hospital and as an inpatient (hospital and nursing home) in past three months, and the frequency of outpatient medical visits in the past three months. 
Despite these limitations, our study was strengthened by proposing the diagnostic weightings of little interest, reduced/ increased sleep, psychomotor retardation/agitation, and concentration problem in the detection of depressive disorder among the elderly patients with chronic physical diseases (DM, COPD/asthma, and CAD). In addition, these findings suggested a sophisticated subscale of the PHQ-9 to detect depressive disorder among the elderly patients with chronic physical diseases, with 4 of cut-off value. Since there is a potential for the false-positive rather than false-negative in the use of our subscale in the PHQ-9, the exclusion procedures including the necessity of melancholia or anhedonia can be additionally elaborated in detecting depressive disorder among the elderly patients with chronic physical diseases.

\section{Acknowledgments}

Our study was supported by the grants from the National Center for Mental Health Research \& Education, the Seoul National Hospital, Republic of Korea and Soonchunhyang University Research Fund.

\section{REFERENCES}

1. Rowe R, Rapaport MH. Classification and treatment of sub-threshold depression. Curr Opin Psychiatry 2006;19:9-13.

2. Park JH, Lee JJ, Lee SB, Huh Y, Choi EA, Youn JC, et al. Prevalence of major depressive disorder and minor depressive disorder in an elderly Korean population: Results from Korean Longitudinal Study on Health and Aging (KLoSHA). J Affect Disord 2010;125:234-240.

3. Koenig HG, Cohen HJ, Blazer DG, Meador KG, Westlund R. A brief depression scale for use in the medically ill. Int J Psychiatry Med 1992; 22:183-195.

4. Steffens DC, Potter GG. Geriatric depression and cognitive impairment. Psychol Med 2008;38:163-175.

5. Cole MG, Dendukuri N. Risk factors for depression among elderly community subjects: a systematic review and meta-analyses. Am J Psychiatry 2003:160:1147-1156.

6. Vink D, Aartsen MJ, Schoevers RA. Risk factors for anxiety and depression in the elderly: a review. J Affect Disord 2008;106:29-44.

7. Himelhoch S, Welle WE, Wu AW, Anderson GF, Copper LA. Chronic medical illness, depression, and use of acute medical service among medicare beneficiaries. Med Care 2004;42:512-521.

8. Massie MJ. Prevalence of depression in patients with cancer. J Natl Cancer Inst Monogr 2004;32:57-71.

9. Musselman DL, Betan E, Larsen H, Phillips LS. Relationship of depression to diabetes types 1 and 2: epidemiology, biology, and treatment. Biol Psychiatry 2003;54:317-329.

10. Rudisch B, Nemeroff CB. Epidemiology of comorbid coronary artery disease and depression. Biol Psychiatry 2003;54:227-240.

11. Van Lieshout RJ, Bienenstock J, MacQueen GM. A review of candidate pathways underlying the association between asthma and major depressive disorder. Psychosom Med 2009;71:187-195.

12. Cruess DG, Evans DL, Repetto MJ, Douglas SD, Petitto JM. Prevalence, diagnosis, and pharmacological treatment of mood disorders in HIV disease. Biol Psychiatry 2003;54:307-316.

13. Robinson RG. Poststroke depression: prevalence, diagnosis, treatment, and disease progression. Biol Psychiatry 2003;54:376-387.

14. Kanner AM. Depression in epilepsy: prevalence, clinical semiology, pathogenic mechanisms, and treatment. Biol Psychiatry 2003;54:388-398.

15. Wallin MT, Wilken JA, Turner AP, Wiliams RM, Kane R. Depression and multiple sclerosis: review of a lethal combination. J Rehabil Res
Dev 2006;43:45-62.

16. Lee HB, Lyketsos CG. Depression and Alzheimer's disease: heterogeneity and related issues. Biol Psychiatry 2003;54:353-362.

17. McDonald WM, Richard IH, DeLong MR. Prevalence, etiology, and treatment of depression in Parkinson's disease. Biol Psychiatry 2003; 54:363-375.

18. Edege LE. Major depression in individuals with chronic medical disorder: prevalence, correlates and association with health resource untilization, lost productivity and functional disability. Gen Hosp Psychiatry 2007;29:409-416.

19. Bogousslavsky J. William Feinberg lecture 2002: Emotions, mood, and behavior after stroke. Stroke 2003;34:1046-1050.

20. Breitbart W, Rosenfeld B, Pessin H, Kaim M, Funesti-Esch J, Galietta $\mathrm{M}$, et al. Depression, hopelessness, and desire for hastened death in terminally ill patients with cancer. JAMA 2000;284:2907-2911.

21. Sung SH, Lee HY, Shim SH, Jeong HY, Choi JS, Lee HS, et al. Survey of depression in the elderly patients with chronic obstructive pulmonary disease. J Korean Neuropsychiatr Assoc 2013;52:163-172.

22. World Health Organization. The International Classification of Diseases and Related Health Problems, 10th revision (ICD-10). Geneva; World Health Organization; 1992.

23. Hamilton M. A rating scale for depression. J Neurol Neurosurg Psychiatry1960;23:56-62.

24. Yi JS, Bae SO, Ahn YM, Park DB, Noh KS, Shin HK, et al. Validity and reliability of the Korean version of the Hamilton Depression Rating Scale (K-HRDS). J Korean Neuropsychiatr Assoc 2005;44:456-465.

25. Zimmerman M, Martinez JH, Young D, Chelminski I, Dalrymple K. Severity classification on the Hamilton Depression Rating Scale. J Affect Disord 2013;150:384-388.

26. Kroenke K, Spitzer RL, Williams JB. The PHQ-9. Validity of a brief depression severity measure. J Gen Intern Med 2001;16:606-613.

27. American Psychiatric Association. Diagnostic and Statistical Manual of Mental Disorders (4th Ed). Washington, DC; American Psychiatric Association; 1994.

28. Park SJ, Choi HY, Kim K, Hong JP. Reliability and validity of the Korean version of the Patient Health Questionnaire-9 (PHQ-9). Anxiety Mood 2010;6:119-124.

29. Han C, Jo SA, Kwak JH, Pae Cu, Steffens D, Jo I, Park MH. Validation of the Patient Health Questionnaire-9 Korean version in the elderly population: the Ansan Geriatric Study. Compr Psychiatry 2008;49:218-223.

30. Morris JC, Heyman A, Mohs RC, Hughes JP, van Belle G, Fillenbaum G, et al. The Consortium to Establish a Registry for Alzheimer's Disease (CERAD). Part I. Clinical and neuropsychological assessment of Alzheimer's disease. Neurology 1989;39:1159-1165.

31. Lee JH, Lee KU, Lee DY, Kim KW, Jhoo JH, Kim JH, et al. Development of the Korean version of the Consortium to Establish a Registry for Alzheimer's Disease Assessment Packet (CERAD-K): clinical and neuropsychological assessment batteries. J Gerontol B Psychol Sci Soc Sci 2002;57:P47-P53.

32. Sundararajan V, Henderson T, Perry C, Muggivan A, Quan H, Ghali WA. New ICD-10 version of the Charlson Comorbidity Index predicted in-hospital mortality. J Clin Epidemiol 2004;57:1288-1294.

33. Kraemer HC. Assessment of $2 \times 2$ associations: generalization of signal detection methodology. Am Statistician 1998;42:37-49.

34. Erdreich LS, Lee ET. A diagnostic interview: the schedule for affective disorders and schizophrenia. Arch Gen Psychiatry 1978;35:837-844.

35. Bagby RM, Ryder AG, Schuller DR, Marshall MB. The Hamilton Depression Rating Scale: has the gold standard become a lead weight? Am J Psychiatry 2004;161:2163-2177.

36. Hermanns B, Kulzer B. Screening in Diabetes Care: Detecting and Managing Depression in Diabetes. In: Mitchell AJ, Coyne JC, Editors. Screening for Depression in Clinical Practice: An Evidence-Based Guide. Oxford: Oxford University Press, 2010, p. 335-346.

37. Mitchell AJ. Overview of Depression Scales and Tools. In: Mitchell AJ, Coyne JC, Editors. Screening for Depression in Clinical Practice: An Ev- 
idence-Based Guide. Oxford; Oxford University Press, 2010, p. 29-56.

38. Hermanns N, Kulzer B, Krichbaum M, Kubiak T, Haak T. Affective and anxiety disorders in a German sample of diabetic patients: Prevalence, comorbidity and risk factors. Diabet Med 2005;22:293-300.

39. Fraure-Smith N, Lesperance F. Depression and anxiety as predictors of 2 -year cardiac events in patients with stable coronary artery disease. Arch Gen Psychiatry 2008;65:62-71.

40. Goldney RD, Ruffin R, Fisher LJ, Wilson DH. Asthma symptoms associated with depression and lower quality of life: a population survey. Med J Aust 2003;178:437-441.

41. Steffen DC. Separating mood disturbance from mild cognitive impairment in geriatric depression. Int Rev Psychiatry 2008;20:374-381.

42. Naarding P, Schoevers RA, Janzing JG, Jonker C, Koudstaal PJ, Beekman AT. A study of symptom profiles of late-life depression: The influ- ence of vascular, degenerative and inflammatory risk-indicators. J Affect Disord 2005;88:155-162.

43. Zimmerman M, Posternak MA, Chelminski I. Is it time to replace the Hamilton Depression Rating Scale as the primary outcome measure in treatment studies of depression? J Clin Psychopharmacol 2005;25:105110.

44. Bech P. The Hamilton disorders. Psychother Psychosom 1993;60:113115.

45. Lichtenberg PA, Marcopulos BA, Steiner DA, Tabscott JA. Comparison of the Hamilton Depression Rating Scale and the Geriatric Depression Scale: detection of depression in dementia patients. Psychol Rep 1992;70:515-521.

46. Koenig HG, Kuchibhatla M. Use of health services by hospitalized medically ill depressed elderly patients. Am J Psychiatry 1998;155:871-877. 